\title{
LAS RELACIONES ENTRE FAMILIAS DE PRISMAS. UNA EXPERIENCIA CON ESTUDIANTES DE MAGISTERIO
}

\author{
GUILLÉN SOLER, GREGORIA \\ Departamento de Didáctica de la Matemática. Universitat de València. 46071 Valencia \\ gregoria.guillen@uv.es
}

\begin{abstract}
SUMMARY
This paper contains a brief description of a research about classification in the context of solid geometry. Models of answers to tasks given by teacher training students of different courses and specialities are shown. To do the tasks it is necessary to identify and enumerate examples of prism's subfamilies; to judge, state and justify relationships between the subfamilies characterised and to represent by means of a diagram those relationships. Errors made by the teacher training students are exposed as well as the difficulties they encountered when doing the tasks after the quadrilateral inclusive classification was studied and different prism's families had been established. The data analysed was obtained from interviews, class-working sessions and written answers to homework activities. The research was carried out with students of Escola de Magisteri Ausiàs March (Teaching School) of the University of Valencia.
\end{abstract}

\section{PRESENTACIÓN}

La actividad de clasificar es una de las características esenciales de cualquier rama del pensamiento humano y, en particular, una actividad fundamental en las matemáticas. Es sabida la gran utilidad que tienen en matemáticas las clasificaciones jerárquicas ${ }^{1}$ y son numerosas las publicaciones relativas al modelo de Van Hiele que han mostrado claramente que algunos estudiantes tienen problemas con ellas (Battista y Clements, 1992; De Villiers, 1987, 1994; De Villiers y Njisane, 1987; Fuys, Geddes y Tischler, 1988; Van Hiele, 1986).

De Villiers (1987, p. 22) indica que «los niños son capaces de comprender a una edad muy temprana inclusiones de clases como «los gatos y los perros son animales»; sin embargo, es, ciertamente, desde el punto de vista psicológico mucho más difícil con figuras geométricas, ya que definir atributos es realmente más sutil y complejo». Este autor concluye que la inclusión de clases entre diferentes tipos de figuras geométricas (por ejemplo, la inclusión de los triángulos equiláteros en los isósceles y la inclusión del cuadrado en los rectángulos, o incluso la inclusión entre diferentes pares de cuadrilá- teros) no conlleva psicológicamente la misma dificultad, aunque la estructura lógica podría ser la misma. En sus experimentaciones verificó que, de todas las tareas propuestas a los niños (identificación de tipos de figuras, uso de terminología geométrica, interpretación de definiciones dadas, argumentos deductivos de un paso, descripción verbal de propiedades de figuras, deducción más larga y clasificación jerárquica de conceptos geométricos), la clasificación jerárquica resultó ser la tarea más difícil. Además en los cursos superiores se mejoraba muy poco en el rendimiento (De Villiers, 1987). Y, dado que la clasificación jerárquica apenas emergía vagamente entre estudiantes que rendían bastante bien en cuestiones que requerían deducción formal, concluyó que, en contra de la teoría de Van Hiele, la clasificación jerárquica no es un prerrequisito para el pensamiento deductivo formal, sino que esta tarea conlleva más dificultad.

Las investigaciones de De Villiers han ido más allá respecto de las del modelo de Van Hiele y han indicado que «varias dificultades que tienen los niños con la inclusión de clases jerárquicas (especialmente los niños 
más mayores) no subyace necesariamente en la lógica de la inclusión como tal, sino que a menudo tiene que ver con el significado de la actividad, lingüístico y funcional: lingüístico en el sentido de interpretar correctamente el lenguaje usado en la inclusión de clases, y funcional en el sentido de entender por qué la clasificación jerárquica es más útil que la clasificación partición» (De Villiers, 1994, p. 17).

La clasificación en el mundo de los poliedros ha sido menos investigada (Guillén, 1991, 1999a, 1999b). Al pasar a tres dimensiones -que es un cambio dramático, en muchos aspectos-, resulta interesante observar la dificultad que conlleva para los estudiantes las relaciones entre familias de sólidos. La clasificación en los poliedros ofrece grandes posibilidades para ello. Por un lado, permite trabajar diferentes clasificaciones particiones, con criterios de diferentes tipos (visuales, cuantitativos, etc.). Además, se pueden establecer clasificaciones particiones con varios criterios conjuntamente y, si bien las clases resultantes son disjuntas, también se pueden considerar las clases que corresponden a uno sólo de los criterios y, entonces, aparecen relaciones de inclusión entre unas y otras (Guillén, 1991). Y también se pueden establecer clasificaciones inclusivas o jerárquicas; por ejemplo, una clasificación de los prismas cuadrangulares (Guillén, 1999b).

En este artículo nos fijamos en algunos problemas ligados a las clasificaciones mencionadas:

- Identificación y enumeración de ejemplos de las clases (familias de sólidos) establecidas.

- Descripción de las clases (familias de sólidos) establecidas.

- Establecimiento y justificación de relaciones entre clases (familias de sólidos).

- Expresión de las relaciones entre clases (familias de sólidos) de diferentes maneras.

- Construcción de diagramas que representan las clasificaciones establecidas ${ }^{2}$.

Vamos a centrarnos especialmente en las relaciones entre familias de prismas, bien en términos de ejemplos, bien teniendo en cuenta sus propiedades. Después de prestar atención a las tareas de identificación y de enumeración de ejemplos de familias de prismas, indicamos modelos de respuestas de estudiantes de la Escola Universitària de Magisteri Ausiàs March $^{3}$ para las tareas de juzgar y de justificar relaciones de inclusión, exclusión o solapamiento entre familias de prismas. También presentamos los errores que cometieron y las dificultades con las que se enfrentaron cuando, una vez tratada la clasificación inclusiva de los cuadriláteros -y después de haber establecido diferentes familias de prismas-, acometimos la tarea de identificar ejemplos de familias contenidas en familias de sólidos, la tarea de juzgar, de enunciar y de justificar las relaciones que existen entre familias de prismas, y la tarea de representar estas relaciones mediante un diagrama.
Las familias de prismas que han sido el soporte para los resultados que presentamos son, por un lado, las que surgieron al trabajar clasificaciones particiones con criterios geométricos que tienen gran componente visual; así establecimos los prismas rectos y oblicuos, convexos y cóncavos. Al considerar criterios que centran la atención en la/ base/s o en las caras laterales, establecimos los prismas triangulares, cuadrangulares, etc.; así como los prismas de bases regulares y los de bases irregulares; los prismas de caras laterales regulares y los que no tienen todas caras laterales regulares. Clasificar con el criterio de regularidad o igualdad de todas las caras nos llevó a los prismas de caras regulares o a los prismas de caras iguales, respectivamente ${ }^{4}$. Dentro de los prismas cuadrangulares centramos la atención en el cubo, romboedro, ortoedro, paralelepípedo, prismas de bases trapecios isósceles, prismas de bases cometas y los prismas de bases trapecios (Anexo I).

\section{ANTECEDENTES}

El estudio que aquí se presenta es parte de un trabajo más amplio (Guillén, 1999a) realizado sobre la aplicación del modelo de Van Hiele a la geometría de los sólidos. Respecto del tema que nos ocupa en este artículo, lo que destacamos del trabajo de Van Hiele (1986) es: $a$ ) por un lado, la necesidad que señala que el estudiante pase un cierto tiempo con los objetos (clases de objetos) antes de empezar a describirlos; $b$ ) por otro lado, que considera que, después de cierto tiempo, los objetos (clases de objetos) pasarán a ser la representación de todas estas propiedades y habrá una equivalencia entre el objeto con un nombre concreto y las propiedades con las que lo describen (p. 168); y $c$ ) que «después de que los estudiantes han examinado el carácter de señal de varias figuras, descubrirán que algunas combinaciones de propiedades producen las figuras deseadas y que otras combinaciones no» (p. 170). También hay que mencionar su advertencia de que «sólo si la red usual (como se enseña en clase) de relaciones se acepta, el cuadrado tiene que entenderse como perteneciente al conjunto de los rombos. Esta aceptación debe ser voluntaria: en nadie es posible forzar una red de relaciones» (p. 50). Asimismo, Freudenthal (1973, pp. 417-418), al hablar sobre deducción, también advierte de que no se debe imponer al estudiante una red de relaciones.

Otras investigaciones realizadas en este marco que suponen un soporte para el trabajo que presentamos aquí son las que han considerado como objetivo evaluar si los estudiantes pueden identificar y explicar relaciones entre subclases de polígonos (cuadriláteros o triángulos); por ejemplo, que todos los rectángulos son paralelogramos o que los triángulos isósceles pueden ser triángulos rectángulos (Battista y Clements, 1992; Fuys, Geddes y Tischler, 1988). Aunque los trabajos mencionados se refieren a clasificaciones de algunos tipos de polígonos, sus resultados o su «modo de hacer» pueden extenderse en algunos casos a la clasificación en el mundo de los poliedros. También hay que considerar que las clasificaciones de los polígonos surgen inmersas en problemas 
de clasificación en el mundo de los poliedros. Centrar la atención en la base de los prismas nos lleva a las clasificaciones de los polígonos, o de diferentes tipos de polígonos, que a su vez generalizamos a los prismas; así establecemos los prismas de bases regulares e irregulares; los prismas triangulares, cuadrangulares, etc. En el apartado de los resultados indicaremos aquéllos que obtenidos en el contexto de la geometría de los sólidos, trabajando con diferentes representaciones físicas de los sólidos, corroboran los resultados conseguidos por investigaciones realizadas en un contexto de geometría plana.

Como referencia para nuestro trabajo destacamos el trabajo de De Villiers $(1987,1994)$, parte del cual se ha realizado en el marco del modelo de Van Hiele, pues ha sido especialmente importante por el análisis teórico que realiza del papel y la función de la clasificación jerárquica en matemáticas. De Villiers (1994) distingue diferentes tipos de clasificación (clasificación jerárquica y clasificación partición; clasificación a priori y clasificación a posteriori) y da breves comentarios para la enseñanza de una clasificación jerárquica de los cuadriláteros. Ya hemos indicado lo que él entiende por clasificación jerárquica y por clasificación partición ${ }^{1}$. Por clasificación a priori quiere decir «que el proceso de generalización y especialización se utilizan deliberadamente para producir nuevos conceptos que se colocan inmediatamente en relaciones jerárquicas o en partición con los otros conceptos existentes. [...] En general, la función más importante de una clasificación a priori es, por tanto, claramente, la de descubrir o crear conceptos nuevos» (p. 14). Ejemplos que indican que aclaran lo que se entiende por clasificación a priori y a posteriori, con respecto a los cuadriláteros son: «Una clasificación $a$ posteriori ocurriría, por ejemplo, si la clasificación de los cuadrados y los rectángulos se considerara después de que se conocieran durante algún tiempo, y sus propiedades se hubieran examinado minuciosamente. Por otro lado, con una clasificación a priori comenzaríamos con el concepto más especial, un cuadrado, y generalizaríamos el rectángulo y el paralelogramo consecutivamente como conceptos nuevos. Por ejemplo, el rectángulo puede generalizarse del cuadrado eliminando la condición de que todos su lados son iguales, pero manteniendo todavía la condición de igualdad de sus ángulos.» (p.14).

Este mismo autor subraya que la clasificación de cualquier conjunto de conceptos no tiene lugar independientemente del proceso de definir y que se debería enfatizar que una definición partición (y clasificación) no es matemáticamente «errónea» simplemente porque sea partición (por supuesto, siempre que contenga toda la información necesaria para que se excluyan todos los no-ejemplos). Añade que algunas veces son necesarias una clasificación partición y sus correspondientes definiciones para distinguir claramente entre conceptos (por ejemplo, cuadrilátero convexo, cóncavo y cruzado, o cometas cóncavas y convexas). También que, cuando se clasifica y se especifica un cuadrilátero dado, la partición es una estrategia espontánea y natural; y que, «dado que la clasificación y sus correspondientes definiciones son arbitrarias y no absolutas, deberíamos conocer que la elección entre una clasificación jerárquica y una partición es a menudo materia de elección personal y de conveniencia» (p. 13).

Pero el trabajo fundamental de De Villiers (1994) es el que da respuesta a la cuestión de por qué preferimos (convencionalmente) la clasificación jerárquica de varios cuadriláteros convexos en vez de una clasificación partición. En este estudio señala las funciones más importantes de la clasificación jerárquica. De las entrevistas individuales con niños y del contexto de la clase encontró que la inclusión de clases entre diferentes tipos de figuras geométricas no conlleva psicológicamente la misma dificultad. Como posibles razones que explican la discrepancia, enumera los prototipos visuales usados como ejemplos, o la manera en la que se han introducido los conceptos (con definición o con ejemplos). También contempla la posibilidad de que las respuestas de los estudiantes sean consecuencia de la naturaleza errónea de las tareas de identificación visual, donde el estudiante podría marcar el ejemplo del cuadrilátero más general sin conocer que la intención de la cuestión era que marcase también los casos particulares. Respecto a la comparación de pares de cuadriláteros, De Villiers (1987) apunta que «parece que la inclusión del cuadrado en el rectángulo es frecuentemente la más difícil» (p. 22). En este contexto de clase, este autor también encontró estudiantes que podían sacar conclusiones correctas aplicando las definiciones que se le daban y hacer inclusiones de clases jerárquicas aplicando estas definiciones, pero que preferían no hacerlo (De Villiers, 1994, p. 17). Y que «algunos estudiantes tenían dificultad con la palabra es en un enunciado como un cuadrado es un rectángulo. Parecieron interpretarlo como que un cuadrado "es equivalente a" o "es el mismo que" un rectángulo, y, por tanto (bastante correctamente), rechazaron el enunciado como ridículo o falso». Concluye que, para que la clasificación jerárquica de los cuadriláteros tenga sentido en el nivel 3 (ordenación), es esencial que haya tenido lugar una negociación apropiada de significado lingüístico y de significado funcional. Comprobó que el uso del adjetivo especial (un cuadrado es un rectángulo especial) ayudó a que algunos estudiantes comprendieran lo que realmente quiere decir que uno es subconjunto del otro. Y también tuvo efecto positivo que se hiciera referencia a situaciones análogas de la vida diaria, donde los objetos pueden verse como conjuntos especiales de otro más grande, por lo que tienen dos «nombres» diferentes. Para una negociación apropiada de significado funcional apunta como necesario que se den suficientes oportunidades y actividades apropiadas para discutir el valor o función de una clasificación jerárquica, para que empiecen a comprender y apreciar la economía de este tipo de clasificaciones (definiciones) y, después de ver su conveniencia, lleguen a aceptarlas.

Trabajos desarrollados desde el punto de vista de la enseñanza que han tenido en cuenta las sugerencias de De Villiers (1994) para trabajar las clasificaciones jerárquicas de los cuadriláteros -y que también hemos tenido como referencia en este estudio- son los de Crainer y Rubenstein (1993) y Maraldo (1980); en las tareas que desarrollamos en clase (ver el apartado siguiente) se ven 
reflejadas algunas cuestiones que ellos investigaron. En estos estudios, para que se discutiera y aceptara el valor o función de la clasificación jerárquica, se dieron a los estudiantes suficientes oportunidades y se propusieron actividades adecuadas. Crainer y Rubenstein (1993) corresponde a un curso de geometría, de un año de duración, con estudiantes de instituto. La clasificación jerárquica de los cuadriláteros la consideraron en diferentes contextos y en tiempos diferentes y la usaron para: a) clasificar teniendo en cuenta las propiedades que se indican; $b$ ) dar nombres a los cuadriláteros menos familiares; $c$ ) enfatizar las relaciones de inclusión que tienen cuadriláteros de diferentes categorías; $d$ ) representar la jerarquía en un diagrama con forma de red, $e$ ) construir definiciones para diferentes categorías de cuadriláteros, mostrando la generalidad como rasgo de la jerarquía; f) desarrollar argumentos sobre las relaciones de inclusión o de solapamiento; y $g$ ) conectar el álgebra con la geometría. Comprobaron que el marco conceptual sustentado por la jerarquía resultó ser económico, en cuanto que no era necesario trabajar mucho para probar propiedades para los cuadriláteros que en el diagrama quedaban colocados los más altos. También verificaron que, en ese nivel, se podían considerar definiciones alternativas para los cuadriláteros y que los estudiantes eran capaces de verificar que probar otras propiedades derivadas podría ser más fácil o más difícil dependiendo de la definición usada. Esto es, se expandía y reforzaba el concepto de la definición de los estudiantes. Maraldo (1980) centró su actividad en trabajar los convenios que hay implícitos en un diagrama de una clasificación jerárquica de los cuadriláteros (representaban los cuadriláteros más generales a la izquierda de los más específicos), diagrama construido a partir de definiciones dadas para los cuadriláteros implicados. Hizo notar que las flechas sirven para indicar algo muy importante en matemáticas: las propiedades de un cuadrilátero específico del diagrama las tienen todos los cuadriláteros de la derecha, conectados con él, pero no necesariamente todos los de la izquierda. Y también se preocupó de que se discutiera y aceptara el valor o función de la clasificación jerárquica.

Como antecedentes para este estudio también cabe mencionar los trabajos realizados por Castelnuovo (1963, 1979) y Fielker $(1983,1986)$ sobre la clasificación de cuadriláteros o hexágonos, desde el punto de vista de la enseñanza. El trabajo de estos autores se ve ampliamente reflejado en el nuestro. En el trabajo de Fielker (1983, 1986) se plantean problemas muy interesantes sobre clasificación, problemas que en nuestras experimentaciones hemos trasladado a las familias de prismas con bases cuadriláteros y hexágonos. Por otro lado, Castelnuovo $(1963,1979)$, con la construcción de los cuadriláteros con varillas (agujeros) que se juntan con chinchetas, proporciona un entorno dinámico en el que los cuadrados surgen como uno de los posibles rombos, el rectángulo como uno de los posibles paralelogramos, etc. Trabajar la clasificación de los prismas cuadrangulares, centrando la atención en la analogía que existe entre elementos del plano y del espacio que comparten relaciones, nos remite a la clasificación de cuadriláteros y al trabajo de Castelnuovo o de Fielker. Centrar la atención en las caras laterales de los prismas, que son paralelogramos, nos lleva a la clasificación de éstos. Y extendiendo «la manera de hacer» de Castelnuovo, con la construcción o generación de modelos de prismas, nos proporcionamos un entorno dinámico para reflejar determinadas relaciones entre familias de prismas. Por ejemplo, a partir de «pulseras» de cuadrados, cerrando por los dos lados con polígonos, obtenemos una familia de prismas, la de caras laterales regulares; en esta familia hay prismas cóncavos y convexos, y los prismas de caras regulares están contenidos en ella. Y cuando generamos prismas a partir del experimento que muestra Castelnuovo (1979), utilizando una «unidad base» formada por dos polígonos que se juntan con gomitas, como se ilustra en la figura 1, los prismas de caras laterales regulares surgen como algunos de los posibles prismas rectos, los prismas de caras regulares como algunos de los posibles prismas de bases regulares, etc.

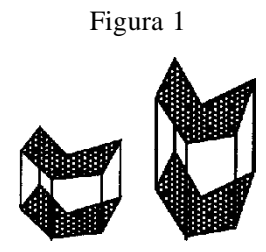

\section{METODOLOGÍA Y CONTEXTO PARA LA EXPERIMENTACIÓN}

El estudio que se describe aquí relativo a la clasificación en el contexto de la geometría de los sólidos fue realizado durante varios cursos académicos (1992-1997). Para obtener la mayor cantidad posible de información sobre lo que aprenden los estudiantes sobre este proceso matemático, utilizamos, por un lado, la entrevista y, por otro, métodos que permiten obtener información de forma simultánea sobre gran variedad de alumnos. Así, realizamos un análisis de:

- Respuestas de diferentes estudiantes, dadas por escrito, a determinadas actividades relativas a la clasificación de familias de sólidos que o bien se plantearon para que se resolvieran en casa antes de tratarlas en clase o se plantearon después de experimentar una unidad de enseñanza.

- Sesiones de trabajo en las que se abordaron problemas ligados a «la clasificación» en el contexto de la geometría.

- Entrevistas individuales a estudiantes que participaron en la experimentación sobre cuestiones relativas a la clasificación en el contexto de la geometría.

\section{Los estudiantes}

El estudio se desarrolló tomando como medio los cursos y grupos de estudiantes de magisterio, de diferentes 


\section{INVESTIGACIÓN DIDÁCTICA}

niveles educativos, que se indican en la tabla I. Los cursos correspondían a asignaturas del currículo en vigor en las que se impartía geometría y de las que la investigadora era profesora. Eran asignaturas que se impartían en el tercer curso, en la especialidad de ciencias $\left(3^{\circ} \mathrm{C}\right)$ o de educación especial $\left(3^{\circ} \mathrm{EE}\right)$, hasta el curso académico 1993-94, o tenían carácter optativo, como Geometría del Espacio (de 4 créditos), del plan de estudios de la Diplomatura de Maestro de la Universitat de València ${ }^{5}$. El número de estudiantes de cada grupo que asistía normalmente a las sesiones oscilaba entre los dos números que indicamos en la tabla I en el apartado «núm. de estudiantes».

Tabla I

Grupos de estudiantes y números entre los que oscila el número de estudiantes por grupo que participaron en las experimentaciones.

\begin{tabular}{|l|c|c|c|}
\hline $\begin{array}{l}\text { GRUPO/S } \\
\mathbf{y} \\
\text { curso/s }\end{array}$ & $\begin{array}{c}\mathbf{3}^{\mathbf{o}} \mathbf{C} \\
\text { (tres grupos) } \\
\mathbf{1 9 9 2 - 9 5}\end{array}$ & $\begin{array}{c}\mathbf{3}^{\mathbf{o} \text { EE }} \\
\text { (dos grupos) } \\
\mathbf{1 9 9 3 - 9 4}\end{array}$ & $\begin{array}{c}\text { Opt. Geom. Esp. } \\
\text { (dos grupos) } \\
\mathbf{1 9 9 4 - 9 6}\end{array}$ \\
\hline $\begin{array}{l}\text { Núm. de } \\
\text { estudiantes/ } \\
\text { grupo }\end{array}$ & $\begin{array}{c}50-60 \\
50-60\end{array}$ & $20-30$ & $8-11$ \\
& $50-60$ & $10-15$ & $5-8$ \\
\hline
\end{tabular}

Previo a la experimentación, los estudiantes de $3^{\circ} \mathrm{C}$ habían cursado una asignatura anual de contenidos matemáticos (la asignatura de $1^{\circ}$ ), una asignatura de resolución de problemas (las matemáticas de $2^{\circ}$ ) y una asignatura de didáctica de las matemáticas. El resto de estudiantes que participaron en la experiencia sólo habían cursado una asignatura anual de contenidos matemáticos (la asignatura de $1^{\circ}$ ). Lo común a todos ellos fue que no habían tratado la geometría de los sólidos anteriormente y habían prestado muy poca atención a la geometría plana.

La intención de seleccionar diferentes grupos era obtener una amplia información sobre procesos de aprendizaje. No pretendía comparar los datos obtenidos en los diferentes grupos.

\section{Sobre las actividades de clase. Procedimiento de ac- tuación}

Las actividades las planteábamos unos días antes de tratarlas en clase para que los estudiantes que quisieran pudieran resolverlas en casa. Tenían como propósito que se abordaran los siguientes problemas, ligados a las clasificaciones particiones o a las clasificaciones inclusivas: $a$ ) identificar y enumerar ejemplos de las familias establecidas al clasificar con un criterio o con varios criterios; en particular, ejemplos de familias que contienen a otras familias de sólidos; $b$ ) enunciar, comprobar o justificar relaciones entre familias de sólidos; y c) reflejar estas relaciones en diagramas que representan las clasificaciones inclusivas (Tablas II, III y IV y diagramas $2 \mathrm{~b}$ y $2 \mathrm{c}$ ).

Las actividades, las planteábamos después de haber tratado una clasificación inclusiva de los cuadriláteros convexos. También se había construido el diagrama que

El diagrama de la figura 2a representa relaciones de inclusión entre los siguientes cuadriláteros: cuadrado (cd), rectángulo (rg), rombo, (rb), paralelogramo (pl), trapecio isósceles (tpi), cometa (ct), trapecio (tp) y cuadrilátero (C).

Las familias de prismas cuadrangulares que se representan en el diagrama 2b son: cubo (C), ortoedro (O), romboedro (R), paralelepípedo (L), prisma de bases cometas (PBc), prisma de bases trapecios isósceles (PBti), prisma de bases trapecios (PBt) y prismas cuadrangulares (PC).

Las familias de sólidos del diagrama 2c son: prisma regular (el cubo) (PReg), prismas de caras regulares, (PCR), prismas de caras iguales (PCI), primas de caras laterales regulares (PCLR), prismas de bases regulares (PBR), primas rectos (PR), prismas convexos (PX) y los prismas (P).

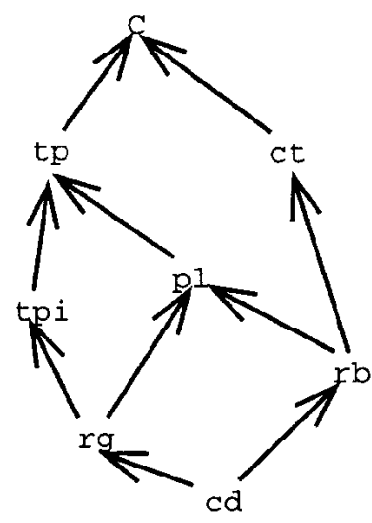

(a)

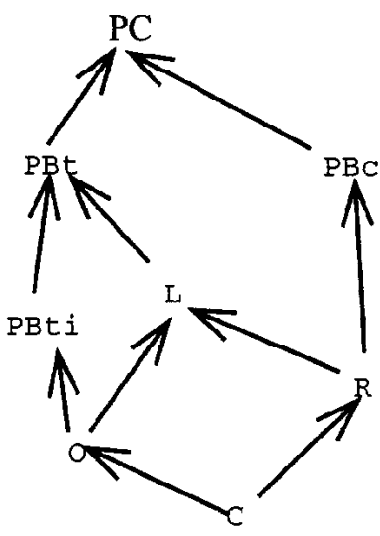

(b)

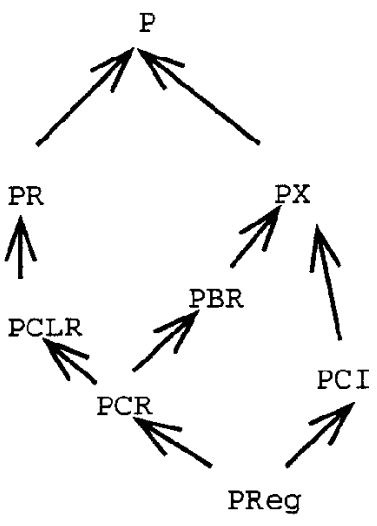

(c) 
reflejaba esta clasificación (Fig. 2a) y se habían establecido las diferentes familias de prismas que servían como soporte para las relaciones que considerábamos, parte de las cuales se muestran en los diagramas $2 \mathrm{~b}$ y $2 \mathrm{c}$. Por ejemplo, 2 b refleja que el ortoedro está contenido en los prismas de bases trapecios isósceles y en los paralelepípedos: «Todos los ortoedros son prismas de bases trapecios isósceles» y «todos los ortoedros son paralelepípedos». También refleja que «los ortoedros son prismas de bases trapecios». Ahora bien, «todos los paralelepípedos no son ortoedros», «todos los paralelepípedos no son romboedros» y de aquí podemos plantearnos seleccionar ejemplos de los paralelepípedos que no son ortoedros, o que no son romboedros, o que no son ni ortoedros ni romboedros. Si nos fijamos en los ortoedros y romboedros, el diagrama muestra que no se puede establecer relación de inclusión en ningún sentido, pero no concluye que estas familias son excluyentes: el cubo está contenido en ambas. Podemos enunciar que «algunos ortoedros son romboedros y otros no lo son» y «algunos romboedros son ortoedros y otros no lo son». Y de aquí cabe plantear: ¿Qué ortoedros no son romboedros? ¿Qué romboedros no son ortoedros?

Al resolver las actividades propuestas, los estudiantes tenían siempre a su disposición modelos de las diferentes familias de prismas tratadas, material comercializado formado por polígonos y diferentes unidades base compuestas por dos polígonos iguales unidos por gomitas (Fig. 1). En sesiones previas se había discutido sobre qué modelos de las diferentes familias podíamos construir con el material comercializado y sobre sus limitaciones. Se habían delimitado familias de prismas de las que podíamos obtener modelos de alguno de sus ejemplos (p.e., de los prismas rectos de bases regulares) y se había hecho notar la conveniencia de recurrir a otros procedimientos para visualizar otros ejemplos de estas familias o de otras como, de prismas oblicuos, de prismas de bases irregulares, de prismas cóncavos. Se aconsejaba a menudo que se recurriera a procedimientos dinámicos que nos permitieran visualizar varios casos; así destacábamos lo que se mantenía y cambiaba en los ejemplos obtenidos o subrayábamos que uno de ellos (el de la familia más específica) era uno de los posibles casos de una familia dada que podíamos generar con el procedimiento utilizado.

Para los paralelepípedos, hacíamos notar que, con material comercializado, se pueden construir los paralelepípedos-ortoedros formados por cuatro rectángulos y dos cuadrados o por seis cuadrados; los romboedros formados por seis rombos-no cuadrados o por seis cuadrados; y también el paralelepípedo formado por cuatro cuadrados y dos rombos-no cuadrados. Ahora bien, destacábamos que con la unidad formada por dos polígonos unidos por gomitas podíamos proporcionarnos representaciones visuales de todo tipo de paralelepípedos. Cuando el problema ya lo tenemos resuelto en el plano, -esto es, aceptamos como posibles paralelogramos el rectángulo-no cuadrado, $\square$, el paralelogramo-no rectángulo-no rombo, $\square$, el rombo-no cuadrado, $\quad$ y el cuadrado, $\square,-$ al cambiar el polígono, eligiendo para la unidad base uno de los posibles paralelogramos, pode-
Tabla II

Tareas de identificación de subfamilias de paralelepípedos.

T.1 Pon ejes de paralelepípedos que además sean romboedros. Haz dibujos de los cuadriláteros que pueden ser caras de esos paralelepípedos. Además, responde a las siguientes preguntas y explica tus respuestas.

¿Las caras de los paralelepípedos que son romboedros pueden ser rectángulos? ¿Pueden ser cualquier tipo de rectángulo? ¿Todas ellas tienen que ser rectángulos? ¿Pueden haber rectángulos de dos tipos?

Responde a las preguntas anteriores para los rombos, en vez de para los rectángulos.

T.2 Repite la tarea T.1 cambiando la expresión «paralelepípedos que además sean romboedros» por «paralelepípedos que no son ortoedros».

Tabla III

Tareas de juzgar y enunciar relaciones entre familias de prismas.

T.3 Indica si las siguientes afirmaciones son correctas o no. Justifica la respuesta.

a) Todos los prismas de caras iguales son prismas convexos, pero todos los prismas convexos no son de caras iguales.

b) $\mathrm{Ni}$ todos los prismas de caras iguales son de caras regulares ni todos los prismas de caras regulares son de caras iguales.

c) No hay ningún prisma que sea a la vez de caras laterales regulares y cóncavo.

T.4 Formula las relaciones que tienen las familias de prismas siguientes y justifica la respuesta.

a) Prismas convexos y prismas de bases regulares.

b) Prismas convexos y prismas de caras iguales.

T.5 Selecciona los términos siempre, a veces o nunca que muestra la relación que hay entre los pares de familias siguientes y justifica la respuesta.

a) Los prismas convexos son siempre, a veces o nunca prismas de caras iguales.

b) Los prismas rectos son siempre, a veces o nunca prismas oblicuos.

mos obtener los romboedros, ortoedros y el cubo. Estos ejemplos de paralelepípedos surgen entre los muchos que se generan por este procedimiento. Y también se puede mostrar que el cubo es uno de los posibles ortoedros con base el cuadrado, lo que cambia en todos estos ortoedros es la altura.

Volviendo a las actividades que planteamos a los estudiantes, vamos a describirlas brevemente.

Identificación y enumeración de ejemplos de clases. En estas tareas se pedía que se identificaran todos los ejemplos de una familia B que contenía a otra familia A; o se pedía que se identificaran ejemplos de B que no fueran ejemplos de A. Los ejemplos y no-ejemplos seleccionados para que se identificaran eran ejemplos de A (que también lo eran de B), ejemplos de B que no lo eran de A y modelos que no eran ejemplos ni de A ni de B. Los presentábamos en diferentes posiciones y con diferentes representaciones físicas (modelos macizos, modelos huecos y como estructuras de aristas). 
Tabla IV

Pares de subfamilias de prismas que se relacionan.

\begin{tabular}{|c|c|}
\hline Tipos de subfamilias & Ejemplo \\
\hline Subfamilias excluyentes establecidas con criterios visuales & Prismas rectos y prismas oblicuos \\
\hline Subfamilias inclusivas & Prismas de caras iguales y prismas convexos \\
\hline $\begin{array}{l}\text { Subfamilias que no son excluyentes ni inclusivas: } \\
\text { Establecidas con un criterio visual. } \\
\text { Criterios de regularidad o igualdad de todas las caras. } \\
\text { Criterios de igualdad o regularidad de parte de las caras. } \\
\\
\text { Criterios de igualdad de aristas o de ángulos. } \\
\text { Una subfamilia establecida con criterio visual y la otra } \\
\text { con criterios de igualdad o regularidad de parte de las caras. }\end{array}$ & $\begin{array}{l}\text { Prismas rectos y prismas convexos. } \\
\text { Prismas de caras iguales y prismas de caras regulares. } \\
\text { Prismas de bases regulares y prismas de caras laterales } \\
\text { regulares. } \\
\text { El romboedro y el ortoedro. } \\
\text { Prismas rectos y prismas de bases regulares. }\end{array}$ \\
\hline
\end{tabular}

Figura 3

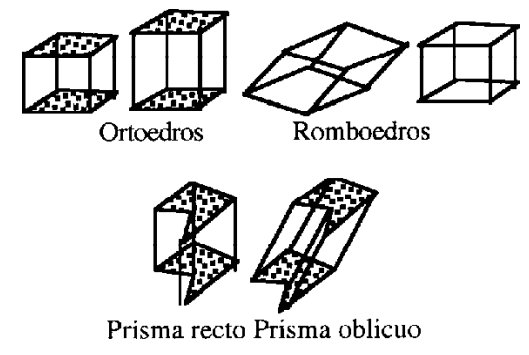

El cubo surge como un posible ejemplo de ortoedro.

Todos los cubos son ortoedros.

Los cubos son ortoedros.

Los cubos son siempre ortoedros

Todos los ortoedros no son romboedros.

Ni todos los ortoedros son romboedros ni todos romboedros son ortoedros.

Los ortoedros pueden ser romboedros.

Los ortoedros son a veces romboedros.

Los prismas rectos nunca son prismas oblicuos.

No hay ningún prisma recto que sea oblicuo.

En otras tareas, lo que se pedía era que se indicasen los ejemplos de estas familias o subfamilias. La tabla II muestra ejemplos de estas actividades que sólo se refieren a paralelepípedos determinados. Para las otras subfamilias de prismas, las actividades que planteamos a los estudiantes eran similares.

Juzgar, enunciar y justificar relaciones entre familias de prismas. Para trabajar este problema ligado a la clasificación, juzgar, enunciar y justificar relaciones entre las clases establecidas, considerábamos situaciones diferentes porque cambiaba la relación que existe entre las familias o porque cambiaba la manera cómo se presentaban las relaciones. La tabla III muestra ejemplos de estas actividades con dos o tres apartados.

Las actividades que planteamos a los estudiantes tenían muchos más apartados que las que indicamos como ejemplo en la tabla III. Las relaciones que se daban enunciadas o los pares de familias que se proponían eran similares a las de la tabla y permitían relacionar otros pares de familias. Nos preocupamos de que se contemplaran las situaciones reflejadas en la tabla IV.
Tabla V

Construcción de diagramas en forma de red para reflejar relaciones entre familias de prismas. Sugerencias.

T.6 Construye el diagrama que relacione las siguientes familias de prismas: prismas rectos, prismas convexos, prismas de bases regulares, prismas de caras laterales regulares, prismas de caras regulares, prismas de caras iguales, prismas regulares (el cubo).

Recuerda lo que ya vimos con la clasificación de los cuadriláteros:

Si dos familias tienen relación de inclusión, en el diagrama que representa la clasificación éstas están conectadas con una flecha que va desde la familia contenida (ahí está el origen) hasta la familia que la contiene (ahí está el extremo).

Los pares de familias que están conectadas por un camino que sigue este esquema $\rightarrow \rightarrow \rightarrow$ tienen relación de inclusión; la familia en la que el camino tiene el origen está contenida en todas las demás del camino, y la familia que hay en el extremo contiene a todas las implicadas en el camino.

Coloca las familias de sólidos de manera que en el primer nivel esté la familia de los prismas; en el segundo nivel, las familias establecidas con criterios visuales; en el tercer nivel, las familias establecidas con criterios que centran la atención en la regularidad de parte de las caras; en el cuarto nivel... 
También tuvimos en cuenta que las relaciones inclusivas se pueden formular como: todos... son..., los... son... y los ... son siempre... Las relaciones excluyentes se pueden verbalizar como: los... nunca son... y no hay ningún... que sea... Y cuando las familias se solapan pero no tienen relación de inclusión podemos indicarlo como: no todos $\operatorname{los} .$. son..., todos... no son... ni todos... son... ni todos... son..., los... pueden ser... y los... son a veces... La figura 3 muestra algunos ejemplos.

En los enunciados de cada tipo nos preocupamos de que se contemplaran ambas posibilidades: que unos fueran correctos y otros no.

La interpretación y la construcción de diagramas en forma de red. Relaciones entre familias de prismas. Otro problema relacionado con la clasificación se refiere a la construcción de diagramas que representan las clasificaciones establecidas. Particularizando las clasificaciones inclusivas, las actividades de este grupo tenían como objeto que se interpretaran los convenios implicados en un diagrama que refleja una clasificación inclusiva de prismas. La tabla $\mathrm{V}$ muestra una actividad de este tipo (Tarea T.6) cuyo diagrama mostramos en la figura 1c. En ella incluimos las sugerencias que dimos para que se tuvieran en cuenta al resolver todas las que planteamos, análogas a la de la tabla, sólo que en la representación estaban implicados otros grupos de familias de prismas.

El proceso de clasificar y el proceso de demostrar. El proceso de clasificar está íntimamente ligado a los procesos de definir y de demostrar. Al fijarnos en la justificación de las respuestas, vale la pena hacer algunos comentarios sobre cuestiones relativas al desarrollo en clase de estas actividades. Por un lado, la tabla VI da cuenta de las justificaciones que hacíamos para explicar el tipo de relación que había entre los pares de familias implicadas en un enunciado. Por otro lado, en clase trabajamos la reformulación de las relaciones entre las clases establecidas en los diferentes tipos de clasificación tratados (clasificaciones particiones y clasificaciones inclusivas). Nos centramos en el uso y negación de los cuantificadores. Trabajamos cómo enunciar las relaciones de diferentes maneras permitía reformular los problemas propuestos y, en algunos casos, poder utilizar lo que ya se había demostrado previamente (al reconvertir una relación en otra que ya se había justificado). En la clase subrayamos además que las relaciones entre dos familias se pueden reformular al seleccionar familias dicotómicas (complementarias) a las consideradas. También abordamos tareas que contenían enunciados con dos relaciones entre clases, por lo que basta con que una de ellas sea falsa para que todo el enunciado lo sea. Dado que algunos estudiantes tenían dificultad para desglosar las dos relaciones que aparecían, consideramos interesante tratar este problema.

\section{Las sesiones de trabajo. Las entrevistas}

Los estudiantes de magisterio del curso 1994-95, de la optativa de Geometría del Espacio estuvieron organiza-
Tabla VI

Desarrollo de algunas pruebas. Secuencia temporal.

Búsqueda de contraejemplo: ejemplo que justifica que las familias no tienen relación de inclusión ni de exclusión. Sugerencias y cuestiones.

Como sugerencia indicamos que había que construir diferentes ejemplos de la primera familia implicada y después cuestionar si entre los ejemplos construidos podía haber ejemplos de la segunda familia y ejemplos de la familia complementaria.

Pruebas para explicar que dos familias tienen relación de inclusión o de exclusión.

- Basadas en ejemplos. Se trabajan las inclusiones o exclusiones de familias en términos de ejemplos.

- En las relaciones de inclusión, en muchos casos se utiliza un procedimiento para generar la familia B que muestra visualmente que la familia A surge entre los ejemplos que se obtienen de la familia B.

- Se verifica que una familia, o un elemento general de ella verifica todas las propiedades (o la definición) de la otra familia. O que no hay ningún elemento de una familia que verifica todas las propiedades de la otra.

- Se aplica en repetidas ocasiones que un ejemplo de una familia tiene que verificar todas sus propiedades y que, si una familia está incluida en otra, verificará sus propiedades.

- Se remarca que entre los grupos de propiedades de las familias hay una relación de inclusión que es la contraria a la que existe entre ellas en términos de ejemplos.

- Se trabajan las inclusiones de familias en términos de grupos de propiedades.

dos en dos grupos durante todo el curso. Las actividades que realizaron en clase (propuestas previamente para que se trabajaran en casa) se grabaron en vídeo o en casete. Cuando los estudiantes ya las habían resuelto las comparaban con las de sus compañeros.

Las entrevistas realizadas en su mayoría fueron individuales, semidirigidas o dirigidas; sólo en algunas los estudiantes intervinieron por parejas. Fueron grabadas en vídeo o en casete. Éstas pretendían aclarar respuestas pobres o respuestas que podían tener interpretaciones diferentes. Por ejemplo, cuando algún estudiante indicaba que los prismas convexos a veces son rectos porque alguno lo es; por ejemplo, el ortoedro. De esta respuesta se puede entender: $a$ ) se piensa que para justificar el «a veces» basta con presentar un ejemplo; o $b$ ) hay un olvido de mostrar el no-ejemplo. Otra respuesta que necesitaría aclaración sería la que en tareas de identificación sólo se indica el ejemplo más general como ejemplo de una familia. La respuesta podría estar basada: $a$ ) en el establecimiento de familias disjuntas; $b$ ) en un no-entendimiento de que la intención de la cuestión era la de seleccionar los casos particulares además de los ejemplos generales; o $c$ ) en los ejemplos prototípicos (en los que se piensa primero para basar sus juicios en ellos).

Otras veces, se buscaba información sobre los diferentes procedimientos que pueden aplicar los estudiantes para resolver determinadas tareas (además del ya utilizado) y 
comprobar si pueden aplicar estos procedimientos en otras actividades. En algunos casos lo que había que averiguar era a qué se debe la respuesta dada: porque se considera que la respuesta dada es suficiente o porque no se puede dar otra. Pongamos por caso cuando se juzga adecuadamente que «los prismas de caras regulares son prismas de bases regulares» y se da una respuesta en términos de ejemplos (se indica que entre los ejemplos de los prismas de bases regulares están los que tienen caras laterales $\square \square$ rectos, más altos y más bajos, y otros oblicuos. Los de caras laterales cuadrados están ahí). Es interesante averiguar qué otro tipo de respuestas se pueden dar: si también se puede razonar en términos de propiedades (por ejemplo, se explica que los primeros cumplen todas las propiedades de los segundos) o en términos de grupos de propiedades (esto es, se explica que el grupo de propiedades de los primeros contiene el grupo de propiedades de los segundos). También es interesante averiguar si se pueden extender estos procedimientos para explicar relaciones entre otros pares de familias de prismas.

\section{ANÁLISIS DE DATOS}

\section{Las respuestas a las actividades}

Cada estudiante de los que participó en la experiencia tenía asignada una carpeta en la que depositábamos las actividades que resolvía por escrito antes de que se abordaran en clase. Además, las respuestas de cada estudiante las agrupábamos según que correspondieran a tareas de identificar subfamilias, de juzgar relaciones, de verbalizarlas o de representar mediante un diagrama las relaciones entre familias. Para cada uno de estos alumnos, y para cada tipo de actividades, en hojas que diseñamos para ello, anotábamos lo que nos parecía que tuviera interés de sus respuestas.

Para cada actividad, las respuestas de varios alumnos, las utilizábamos en plan exploratorio; servían además para corroborar lo que ya habíamos observado los años anteriores con las experiencias previas realizadas con otros cursos. Como ejemplo, en la tabla VII, mostramos las características que delimitamos para hacer observaciones en las respuestas de los estudiantes que habían construido diagramas.

Con las características observadas, diseñamos láminas para poder registrar si otros estudiantes presentaban las características delimitadas. Así decidimos si éstas se presentaban con la suficiente frecuencia para poder presentarla como resultado de la investigación. Si con las respuestas de estos estudiantes aparecía algo nuevo que merecía atención, se introducía también como característica para corroborar con las respuestas de estudiantes que aún no se habían analizado. Para cada actividad los encabezamientos de las láminas hacían referencia a las características subrayadas sobre ella y a los estudiantes que la habían resuelto. En las casillas indicábamos el número de «errores» que presentaba el estudiante correspondiente y, en una lectura posterior, nos fijábamos en los pares de familias que estaban implicados en ellos. Todos los datos que se repitieron en todos los grupos de magisterio que participaron en la investigación, y que se reflejaban en más de 2 o 4 estudiantes, según el número de estudiantes del grupo, los presentamos en el apartado siguiente como resultados de la investigación.

\section{Tabla VII}

Características a destacar en la construcción de diagramas que representan relaciones de familias de prismas.

a) No se dan a las flechas el significado que se ha asignado por convenio.

b) Hay familias que no están colocadas en el nivel adecuado.

c) Se relacionan familias que no tienen relación de inclusión.

d) Se relacionan familias en sentido contrario a la relación de inclusión que presentan: el origen de la flecha está en la familia que debería estar en el extremo.

e) No se relacionan familias que sí que presentan relación de inclusión en un sentido (faltan por poner flechas que conectan determinadas familias).

f) Se conectan directamente familias para las que ya queda reflejada su relación de inclusión a través de un camino, que relaciona las familias implicadas con otras.

Respecto de la identificación de ejemplos de las subfamilias, anotamos los que no se identificaban adecuadamente y tratamos de averiguar (a partir de lo que se había escrito al explicar la respuesta o mediante entrevistas) si la exclusión de las familias específicas como ejemplos de las más generales se debía a que se hacían clasificaciones particiones o a otras razones. El resultado 1 que presentamos en el apartado siguiente corresponde a estas observaciones. Para las tareas en las que se había que enumerar ejemplos de subfamilias que cumplían ciertas condiciones (por ejemplo, que no fueran romboedros) y en las que se preguntaba sobre el tipo de polígonos de sus caras, anotamos los ejemplos que se indicaban y no lo eran, y los que no se señalaban y sí lo eran. También nos fijamos en si se tenían en cuenta las condiciones impuestas o si se indicaban simplemente los ejemplos de la familia general implicada en la actividad. Pongamos por caso, aunque se preguntaba por los paralelepípedos que no fueran romboedros, algunos estudiantes reflejaban con su respuesta que no tenían en cuenta la condición: indicaban los ejemplos que había de paralelepípedos, y al hablar sobre el tipo de caras, respondían de la misma manera. Otras respuestas reflejaban que no se entendía la condición que se imponía, o no se entendían los cuantificadores implicados en las preguntas que hacían referencia al tipo de caras. Cuando se pedía que se indicasen ejemplos de paralelepípedos que no fueran ortoedros se indicaba: las caras sólo pueden ser rectángulos, cuadrados también pueden ser, el cubo es ortoedro. Otros estudiantes respondieron: no puede haber ningún rectángulo, porque sino sería ortoedro. Los resultados 2 y 3 dan cuenta de estas observaciones. 
La interpretación que hacíamos de las respuestas dadas para las tareas en las que se han de juzgar, verbalizar y representar relaciones entre familias de prismas nos llevó a precisar observaciones que se referían especialmente a modelos de respuesta para justificar relaciones entre familias de sólidos. Detallamos también dificultades que conllevan estas tareas, las cuales conducen a que los estudiantes cometan determinados errores. Así, después de contrastar con entrevistas individuales las respuestas que considerábamos ambiguas, y de corroborar cada observación con respuestas de varios estudiantes de los diferentes grupos en los que realizamos la experiencia, delimitamos: $a$ ) modelos de respuestas; $b$ ) tipos de relaciones entre familias que presentan más o menos dificultad; c) los enunciados que dificultaban o facilitaban las tareas; y $d$ ) otras dificultades con las que se enfrentaban los estudiantes, que permiten graduar el nivel de dificultad de la tarea. Los seis resultados numerados del 4 al 9 dan cuenta de ello.

\section{Las sesiones de trabajo y las entrevistas}

Para los datos recopilados en cintas de vídeo o de casete, hicimos transcripciones literales (para algunas entrevistas) o resúmenes (para las sesiones de clase del grupo de Geometría del Espacio) que contenían lo destacable de cada sesión. Así pues, conseguimos varios protocolos que constituían el objeto de análisis, y también varios resúmenes, en los que apuntamos lo que se consideraba relevante, bien porque confirmaba lo que ya se había observado con las experimentaciones con otros estudiantes, bien porque podía introducirse como algo nuevo que tenía que ser objeto de experimentación.

Como ejemplo de entrevista estructurada cabe señalar la que utilizamos para aclarar respuestas que se habían dado en tareas de identificación de subfamilias, y que mostramos en el anexo II, con la intención de que se llegase a establecer una clasificación jerárquica en los paralelepípedos y que se llegase a representar la clasificación en un diagrama. El guión de esta entrevista es una adaptación para los paralelepípedos del que se presenta en De Villiers (1987) para la identificación de paralelogramos.

\section{RESULTADOS: MODELOS DE RESPUES- TAS. DIFICULTADES Y ERRORES}

Vamos a comenzar presentando tres resultados que hacen referencia a identificación y enumeración de ejemplos de clases (familias de prismas) establecidas con clasificaciones inclusivas, centrando la atención en si las respuestas de los estudiantes reflejan dificultades de la tarea asignada. Considerando A y B como familias de prismas, de manera que $\mathrm{B}$ contiene a $\mathrm{A}$, el resultado $1 \mathrm{da}$ cuenta de la identificación de ejemplos de $\mathrm{B}$ cuando se muestran modelos que son ejemplos de A, ejemplos de By $\neg$ A y no ejemplos de B. Los resultados 2 y 3 se refieren a la enumeración y descripción de ejemplos de clases (familias de prismas) de la forma B $\mathrm{y} \neg \mathrm{A}$.
Continuamos exponiendo los seis resultados que intentan dar cuenta de lo que hicieron los estudiantes que participaron en la experimentación cuando se les plantearon tareas de juzgar, enunciar, representar y justificar relaciones entre clases (familias de sólidos) y cuando se establecieron clasificaciones inclusivas: ¿Qué modelos de respuesta dieron? ¿Con qué dificultades se encontraron? ¿Qué errores cometieron.

1) En tareas de identificación de ejemplos de familias de prismas establecidas con clasificaciones inclusivas, a menudo las respuestas de los estudiantes son consecuencia de:

- La familiaridad que se tiene con los ejemplos; en algunos casos no se incluyen como ejemplos los que pertenecen a una subfamilia contenida porque en estos ejemplos no se suele pensar (aunque se acepte luego que son ejemplos).

- La naturaleza errónea de las tareas de identificación visual, en las que el estudiante selecciona el ejemplo de la familia de prisma más general sin conocer que la intención de la cuestión puede ser que se seleccionen también los casos particulares.

- Las respuestas son consecuencia de que los estudiantes establecen clasificaciones particiones.

- Algunos estudiantes que pueden sacar conclusiones correctas sobre inclusiones de familias en términos de ejemplos, o aplicando definiciones, prefieren no hacerlo.

Por lo tanto, si no se realizan entrevistas de las respuestas de los estudiantes a tareas concretas, no siempre se pueden sacar conclusiones respecto de si los estudiantes realizan clasificaciones particiones cuando se proponen clasificaciones inclusivas, o respecto de si los estudiantes realizan clasificaciones particiones en lugar de clasificaciones inclusivas porque las primeras conllevan menos dificultad.

Este primer resultado presenta cuatro razones que explican la identificación de ejemplos de subfamilias que realizan los estudiantes. La primera razón queda reflejada en las investigaciones sobre polígonos de Hershkowitz (1990); ella lo llama fenómeno prototipo y juicios prototípicos. Las tres razones siguientes podemos encontrarlas en De Villiers (1994), y las dos últimas en Battista y Clements (1992).

2) Las tareas de mostrar ejemplos de familias de prismas de la forma B y A o By $\neg$ A (que cumplen determinadas condiciones) presentaron grandes dificultades para algunos estudiantes. A menudo no tenían en cuenta la condición impuesta o se tenía en cuenta pero no se podía aplicar correctamente. Por ejemplo, se intercambiaba el papel de las dos familias implicadas en el enunciado.

La explicación podemos encontrarla en el propio contenido del concepto. Aun razonando en términos de ejemplos, que conlleva menos dificultad que razonar en términos de propiedades, se tienen que considerar los 
ejemplos de $\mathrm{B}$ distribuidos en dos familias (ByA y $\mathrm{By} \neg \mathrm{A})$; se tiene que aplicar que si $\mathrm{A} \supset \mathrm{B}$ los ejemplos de AyB son los de A, o que los ejemplos de By $\neg A$ son los de $\neg A$ cuando se toma como referencia $B$.

3) En la tarea de mostrar ejemplos de subfamilias de prismas que cumplen determinadas condiciones, cuando se pregunta por el tipo de caras que pueden tener y si todas ellas pueden ser de una determinada clase, a menudo las respuestas de los estudiantes reflejan que no pueden usar de manera matemáticamente correcta los cuantificadores implicados en las cuestiones y que no expresan correctamente la negación de estos cuantificadores. Por ejemplo, no tener todas las caras regulares se indica en el sentido de que no puede tener ninguna cara regular; o no tener todas las caras de un tipo se expresa como que no puede tener ninguna cara de ese tipo.

Asimismo, las respuestas también reflejan dificultades para negar criterios utilizados para establecer clases en las que hay implicadas varias propiedades. Por ejemplo, para los prismas de bases irregulares se indica que las bases tienen lados y ángulos distintos.

Estos errores podemos considerarlos como la manifestación de las dificultades cognitivas que impiden comprender cómo usar y negar los cuantificadores.

4) En tareas en las que se han de juzgar, verbalizar, representar y descodificar relaciones entre familias de prismas hay respuestas incorrectas que cabe comentar:

- En tareas en las que se formula una relación entre las subfamilias A y B, algunos estudiantes consideran la relación inversa. Esto es, no se juzga la relación entre las familias A y B sino la que existe entre B y A.

- En un diagrama no se interpreta adecuadamente el significado de dos familias que están conectadas con una flecha. Se muestran errores al enunciar relaciones entre familias.

- En un diagrama no se interpreta adecuadamente el significado de dos familias que no están conectadas con una flecha. Es usual que se interpreten como familias disjuntas (no tienen ejemplos comunes).

- Entre los diagramas que construyen los estudiantes que representan una clasificación de los prismas cuadrangulares es usual encontrar algunos que no pueden considerarse ni representaciones de clasificaciones del plano ni del espacio, porque en ellos hay nombres de sólidos y de polígonos.

\section{Sobre dificultades remarcamos:}

- Algunos estudiantes tienen dificultades para establecer, representar e interpretar si entre dos familias de sólidos dadas hay relación de inclusión, de exclusión, o tienen intersección pero no están incluidas una en otra.

- Algunos estudiantes tienen dificultad con la palabra es en un enunciado como un cubo es un ortoedro.
Cabe señalar que la segunda dificultad que indicamos en este resultado fue puesta de manifiesto en su totalidad por De Villiers (1994), en el contexto de la clasificación de cuadriláteros.

5) Modelo de respuesta: Para justificar relaciones de inclusión, exclusión o solapamiento entre clases (familias de sólidos) se indican ejemplos o contraejemplos, o bien ejemplos y contraejemplos. Se indican nombres de familias o dibujos de alguno de sus ejemplos. O se indican las propiedades que tienen que cumplir los ejemplos y contraejemplos. En algunos casos sólo se indican las propiedades del polígono de las bases del prisma.

En algunas respuestas, los ejemplos o contraejemplos dados son adecuados y se explica la respuesta sobre por qué las familias seleccionadas son ejemplos o contraejemplos, o cómo se delimitan éstos. En otras, se dan los contraejemplos sin más explicaciones. A veces se hace referencia a algunas propiedades del ejemplo o contraejemplo pero no se precisan lo suficiente.

En algunas respuestas incorrectas:

- No se razona la relación que se propone sino la inversa.

- Se indica como contraejemplo un prisma que no es ejemplo de ninguna de las familias de prismas que se consideran.

- Al explicar con un contraejemplo que éste es ejemplo de una familia pero no ejemplo de la otra, a veces se confunde el papel de las familias y se señalan propiedades que no son las adecuadas para explicar la respuesta.

- Se razona con un ejemplo, o con un número insuficiente, relaciones de inclusión entre familias. Esto es, para las relaciones que una respuesta matemáticamente completa exige de demostraciones o de verificar que una familia cumple la definición de otra, en algunos casos se dan ejemplos o contraejemplos (adecuados o no) y se indican algunas propiedades de ellos.

Sobre dificultades cabe señalar:

- Justificar si la relación que se enuncia es correcta presenta grandes dificultades para los estudiantes. En general, justificar que dos familias se solapan pero no están incluidas una en otra presenta menos dificultades para los estudiantes que justificar una relación de inclusión entre familias, a no ser que éstas sean muy conocidas para los estudiantes y estén muy acostumbrados a ver una como ejemplo de la otra.

- Ahora bien, seleccionar el ejemplo de la familia correspondiente que pueda servir de contraejemplo y explicar que es ejemplo y no-ejemplo de las familias implicadas también conlleva dificultad para los estudiantes.

- Las relaciones de la forma no puede haber... que no sean... conllevan más dificultad para los estudiantes que las relaciones enunciadas de otras maneras. 
6) Modelo de respuesta: Tanto en los enunciados para los que una respuesta adecuada es a partir de ejemplos o contraejemplos como en los que hay que seleccionar la palabra siempre o la palabra nunca, a veces se explica la respuesta verificando que una familia cumple (o no cumple) la definición o las propiedades de la otra (que en algunos casos puede bastar con una propiedad).

En algunas respuestas incorrectas:

- No se razona la relación que se propone sino la inversa.

- Se razona con un número insuficiente de propiedades que una familia A está incluida en otra familia B.

- Para explicar que dos familias A y B son excluyentes se razona que una subfamilia de A no verifica todas las propiedades de $\mathrm{B}$.

Sobre dificultades cabe destacar:

- La dificultad que conlleva seleccionar las propiedades que caracterizan a una familia depende de la familia considerada.

- Considerar los ejemplos generales de las familias de prismas (por ejemplo, el prisma n-agonal de caras regulares) y razonar a partir de ellos si verifican o no las propiedades de otras familias conllevan también dificultades para los estudiantes.

Asimismo, resulta difícil para los estudiantes distinguir cuando en un razonamiento basado en un ejemplo concreto se han utilizado las características propias del ejemplo, o no ha sido así, en cuyo caso este ejemplo puede utilizarse como representante de la familia y el razonamiento sería válido en general.

7) Modelo de respuesta: Para explicar relaciones de inclusión entre familias se razona en términos de inclusiones de los ejemplos de ellas o en términos de inclusiones de grupos de propiedades.

En algunas respuestas incorrectas:

- No se relacionan adecuadamente las familias implicadas y sus grupos de propiedades.

- Se asocian con relación de inclusión algunas familias que no tienen esta relación y, para otras que la tienen, seleccionan la partícula $a$ veces.

Sobre dificultades cabe indicar:

- Considerar las familias en términos de ejemplos, esto es, divididas en subfamilias en las que se incluyen parte de los ejemplos de la familia más general, facilita que los estudiantes puedan establecer relaciones de inclusión entre clases (familias de primas), si bien esta tarea también conlleva dificultades para los estudiantes.

- Llegar a ver las relaciones de inclusión entre familias como la inclusión inversa de sus grupos de propiedades y aplicar correctamente relaciones entre familias conlleva gran dificultad para los estudiantes.

8) Modelo de respuesta: Para explicar relaciones de exclusión entre familias se razona en términos de grupos de propiedades.

Algunas respuestas incorrectas reflejan un no-entendimiento de los cuantificadores implicados en las cuestiones y que no se expresa aún de manera correcta la negación de dichos cuantificadores. Como en las respuestas del resultado 3, verificamos de nuevo que no tener todas las caras regulares se expresa en el sentido de que no puede tener ninguna cara regular; no tener todas las caras laterales rectángulos se indica como que no tiene ninguna cara lateral rectángulo o que el polígono irregular tiene lados y ángulos distintos.

Sobre dificultades subrayamos que justificar relaciones de exclusión entre familias dicotómicas establecidas con criterios visuales conlleva menos dificultades que considerar familias dicotómicas establecidas con criterios que centran la atención en la regularidad o igualdad de parte de las caras (las bases o las caras laterales) o de todas ellas.

De nuevo la justificación la encontramos en que en este último caso hay que negar un criterio que afecta a parte de las caras o a todas ellas y además en el criterio puede haber implicadas varias propiedades.

Los resultados obtenidos relativos a «errores» de los estudiantes y a las dificultades detectadas para determinados tipos de relaciones pueden explicarse desde el contenido matemático implicado en ellas. Ésta puede ser la explicación de los resultados 5, 6,7 y 8. Cuando se está familiarizado con los ejemplos de una familia, justificar que se selecciona el término a veces -esto es, buscar el ejemplo y el contraejemplo, y verificar que lo sonconlleva menos dificultad que explicar la respuesta cuando se selecciona el término siempre. En este caso, para una respuesta correcta y completa, más que trabajar con ejemplos (en cuyo caso se ha de considerar uno general que represente a todos, y para él verificar todas las propiedades imprescindibles de la otra familia), hay que delimitar las propiedades que caracterizan a la primera familia (la que aparece a la izquierda) y observar que contienen todas las propiedades que caracterizan a la otra (la que aparece a la derecha). Para una respuesta completa, se tienen que considerar todas las propiedades imprescindibles de cada una de las familias implicadas.

Cuando se selecciona el término nunca, para una respuesta correcta y completa necesariamente se han de considerar todos los ejemplos de una de las familias que se relacionan. Y en relaciones de este tipo, en muchos casos hay que distinguir unos ejemplos de otros porque dejan de verificar diferentes condiciones de la definición de la otra familia.

Un caso particular que podemos examinar es cuando se comparan dos familias dicotómicas en las que pueden 
venir caracterizadas por una única propiedad. En estos casos es más inmediato verificar que ningún ejemplo de una de las familias (la que aparece a la izquierda) verifica todas las propiedades imprescindibles de la otra (la que aparece a la derecha), dado que para ellas se puede considerar un ejemplo genérico para explicarlo. Se puede explicar a partir de él que no puede verificar las propiedades que caracterizan a la otra familia y, para ello no se utilizan las propiedades específicas del ejemplo, por lo que la explicación puede servir para cualquier ejemplo.

9) Modelo de respuesta: Para explicar que se selecciona el siempre o el nunca a veces se utiliza la familia complementaria de una de las que aparece en la relación dada.

Los resultados anteriores son coherentes con los que se presentan en otras publicaciones relativas al modelo de Van Hiele en cuanto que todos ellos subrayan la dificultad que conllevan las clasificaciones jerárquicas. Los resultados de este trabajo perfilan lo que puede ocurrir cuando los estudiantes se enfrentan ante las clasificaciones jerárquicas y la deducción. Como en De Villiers (1987) y De Villiers y Njisane (1987), de ellos se desprende que la inclusión de clases entre diferentes figuras geométricas no conlleva la misma dificultad, aunque la estructura lógica sea la misma. Al igual que estos autores, nos atrevemos a conjeturar que la clasificación jerárquica puede resultar una tarea más difícil que las deducciones de un sólo paso, por lo que esta tarea no es un prerequisito para el pensamiento deductivo formal, sino que conlleva más dificultad.

\section{IMPLICACIONES DIDÁCTICAS}

Los hallazgos de este estudio tienen implicaciones prácticas tanto para maestros o profesores como para diseñadores de currículo. Pueden utilizarse para el diseño de actividades que tengan como propósito la enseñanzaaprendizaje de los sólidos y de ellos se desprenden también sugerencias para la instrucción.

1) Castelnuovo (1963, 1979) utiliza las varillas con agujeritos y chinchetas para juntarlas, permitiendo así que algunos cuadriláteros se transformen fácilmente en otros. Nuestras experimentaciones han confirmado la gran utilidad que tiene este material usado por Castelnuovo, así como los entornos dinámicos que proporcionan los procedimientos de construir o generar modelos de familias de sólidos para que algunos estudiantes vean y acepten la posibilidad de inclusiones jerárquicas.

2) Señalamos la conveniencia de que los estudiantes puedan trabajar, con diferentes actividades y en diferentes tiempos, las relaciones entre familias de sólidos. Como hemos subrayado, los estudiantes tienen dificultades para juzgar, enunciar y justificar si entre dos familias de sólidos hay relación de inclusión, de exclusión o tienen intersección pero no están incluidas una en otra. La construcción de diagramas que muestran las relaciones entre familias también conlleva dificultad. Hay que dedicar tiempo y atención para que los estudiantes puedan salvar estas dificultades, que les pueden llevar a cometer los errores subrayados en este trabajo.

3) Dado que justificar las relaciones de inclusión o de exclusión que existen entre familias de sólidos conlleva más dificultad para los estudiantes que justificar una relación de solapamiento, sugerimos que en primer lugar se justifique que dos familias se solapan pero no están incluidas una en otra. Hacemos notar la conveniencia de trabajar la búsqueda de ejemplos y no-ejemplos para justificar estas relaciones de solapamiento.

Demostrar relaciones de inclusión entre familias conlleva grandes dificultades. Proponemos que se trabajen en primer lugar estas inclusiones en términos de ejemplos, para aplicar luego que, si una familia está incluida en otra, se verificarán sus propiedades. Por último se aplicará que entre los grupos de propiedades de las familias hay una relación de inclusión que es la contraria a la que existe entre ellas en términos de ejemplos.

4) Es aconsejable usar el adjetivo especial para reflejar algunas relaciones de inclusión (por ejemplo, un cubo es un ortoedro especial). Al igual que De Villiers (1994), comprobamos que ayuda a que algunos estudiantes comprendan lo que realmente quiere decir que uno es subconjunto del otro. También tuvo efecto positivo hacer referencia a situaciones análogas de la vida diaria,en las que los objetos pueden verse como conjuntos especiales de otro más grande, por lo que tienen dos «nombres» diferentes (De Villiers, 1994).

5) Subrayamos la conveniencia de enunciar las relaciones existentes entre las familias de varias maneras que son equivalentes, tanto si son relaciones de inclusión, exclusión o solapamiento. Aconsejamos que se haga notar y se aplique que la relación de inclusión entre familias se puede formular como: todos... son..., los... son..., los... son siempre..., no hay ningún elemento... que no sea... Las relaciones de exclusión se pueden enunciar como: los... nunca son... y no hay ningún... que sea... Y las relaciones de solapamiento como: no todos los... son..., todos... no son..., ni todos... son... ni todos... son..., los... pueden ser... y los... son a veces...

Al reformular las relaciones propuestas se puede averiguar si dos enunciados son equivalentes o no, o bien si la reformulación puede tener como objeto convertir un enunciado dado en otro, para el que ya tengamos una demostración realizada, o cuya demostración del enunciado equivalente resulta más sencilla.

6) Hay que prestar atención a determinar el sentido de la relación que se enuncia. Consideramos interesante averiguar si, ante un enunciado de una relación, se juzga la relación entre las familias A y B o la que existe entre B y A. Este problema hay que abordarlo en clase para evitar respuestas muy comunes en las que, si bien en la relación que se da se formula una relación entre las familias A y B, para explicar las respuestas se considera la inversa. 
7) También vamos a subrayar los enunciados en los que hay implicadas dos relaciones entre familias. Sugerimos que se incluyan en las tareas alguno de ellos, ya que, entre otros, permiten abordar los siguientes problemas: a) delimitar las dos relaciones implicadas en cada enunciado; y $b$ ) discutir que los enunciados sólo son correctos cuando las dos relaciones que figuran en ellas son correctas, ya que si, una de las dos relaciones que hay en cada actividad es falsa, todo el enunciado completo es falso al serlo una de sus partes.

8) Señalamos lo interesante que resulta tratar la clasificación de los prismas cuadrangulares una vez que se han tratado clasificaciones de los cuadriláteros. Esta tarea permite mostrar cómo se puede aplicar la analogía para explicar las relaciones de inclusión o no-inclusión que hay entre pares de familias de prismas cuadrangulares (Guillén, 1999b). Se puede hacer ver que el problema puede resolverse trasladando el problema al plano y justificando ahí la respuesta, una vez que se hayan hecho las traducciones correspondientes de los elementos análogos. Así, se plantea realmente el problema de hallar relaciones entre el cuadrado y el rectángulo, el rombo y el rectángulo, el paralelogramo y la cometa, porque luego vamos a extenderlas a los elementos análogos en el espacio.

Queremos advertir de dos problemas que se presentan a menudo, que son propios de esta clasificación: uno se refiere al peligro que conlleva aprenderse los diagramas de memoria; y otro es un problema de lenguaje, provocado por tener que distinguir entre nombres que corres- ponden a familias de polígonos y otros que corresponden a sólidos. Es fundamental prestar atención especial a estos problemas cuando se resuelven estas actividades.

\section{NOTAS}

${ }^{1}$ Al igual que De Villiers (1994), por clasificación jerárquica queremos decir la clasificación de un conjunto de conceptos de tal manera que los conceptos particulares forman subconjuntos de los más generales. Las clasificaciones inclusivas o jerárquicas provienen de las relaciones de orden. En el ejemplo que damos de la clasificación y ordenación jerárquica de familias de prismas la relación es de orden parcial, sin embargo, en una clasificación-partición, los subconjuntos son disjuntos unos de otros; la clasificación partición se establece a partir de relaciones de equivalencia.

${ }^{2}$ Como hemos indicado en la nota 1 , en las clasificaciones inclusivas o jerárquicas, las inclusiones entre clases son el aspecto fundamental. Estas clasificaciones se pueden representar mediante un modelo que es una red.

${ }^{3}$ En esta escuela universitaria es donde se prepara a los futuros profesores de educación infantil (hasta 5 años) y primaria (612 años).

${ }^{4}$ Hemos nombrado como prismas de caras regulares la familia de los prismas que tienen bases regulares y caras laterales cuadrados. Los prismas de caras iguales son los romboedros (tienen caras rombos) y hemos considerado el cubo como ejemplo de romboedro

${ }^{5}$ Esta asignatura corresponde a una versión del curso de geometría impartido a los estudiantes de $3^{\circ} \mathrm{C}$ adaptado a las nuevas circunstancias.

\section{REFERENCIAS BIBLIOGRÁFICAS}

BATTISTA, M.T. y CLEMENTS (1992). Students' Cognitive Construction of Squares and Rectangles in Logo Geometry, en Geeslin, W. y Graham, K. (eds.) (1992). Proceedings of the $8^{\text {th }}$ International Conference of the International Group for the Psychology of Mathematics Education, 1, pp. 57-64. New Hampshire: Durham.

CASTELNUOVO, E. (1963). Didactica della Matematica Moderna. Florencia: La Nuova Italia. Trad. cast. 1970. Didáctica de la matemática moderna. México: Trillas.

CASTELNUOVO, E. (1979). La Matematica. La Geometria. Trad. cat.: La matematica. La geometria. 1981. Barcelona: Ketres.
CRAINE, T.V. y RUBENSTEIN, R.N. (1993). A Quadrilateral Hierarchy to Facilitate Learning in Geometry. The Mathematics Teacher, 86(1), pp. 30-36.

DE VILLIERS, M.D. (1987). Research Evidence on Hierarchical Thinking, Teaching Strategies and the Van Hiele Theory: Some Critical Comments. Research Unit for Mathematics Education (Rumeus), 10. Stellenbosch: South Africa.

DE VILLIERS, M. D. y NJISANE, R. M. (1987). The Development of Geometric Thinking among Black High School Pupils in Kwazulu (Republic of South Africa), en Bergeron, J.C., Herscovics, N. y Kieran, C. (eds.) (1987). Proceedings of the $11^{\text {th }}$ International Conference for the International Group 
for the Psychology of Mathematics Education, 3, pp. 117123. Montreal: Canadá.

DE VILLIERS, M.D. (1994). The Role and Function of a Hierarchical Classification of Quadrilaterals. For the Learning of Mathematics, 14(1), pp. 11-18.

FIELKER, D.S. (1981-1983). Removing the Shackles of Euclid, Mathematics Teaching, núms. 95-104. Trad. caste. 1987. Rompiendo las cadenas de Euclides. Madrid: Ministerio de Educación y Ciencia.

FIELKER, D.S. (1986). Hexágonos. Epsilon, 8, pp. 5-10.

FREUDENTHAL, H. (1973). Mathematics as an Educational Task. Dordrecht: D. Reidel.

FUYS, D., GEDDES, D. y TISCHLER, R. (1988). The Van Hiele Model of Thinking in Geometry among Adolescents. Journalfor Research in Mathematics Education Monograph, 3. NCTM: Reston.

GUILLÉN, G. (1991). El mundo de los poliedros. Madrid: Síntesis.

[Artículo recibido en julio de 2000 y aceptado en abril de 2001.]
GUILLÉN, G. (1999a). El modelo de Van Hiele aplicado a la geometría de los sólidos. Observación de procesos de aprendizaje. Colección: Tesis doctorales en microfichas. Valencia: Universidad de Valencia.

GUILLÉN, G. (1999b). Una clasificación inclusiva de prismas cuadrangulares. Dificultades. Actas de las IX JAEM. Lugo. Septiembre de 1999, pp. 535-537.

GUILLÉN, G. (2000). Sobre el aprendizaje de conceptos geométricos relativos a los sólidos. Ideas erróneas. Enseñanza de las Ciencias, 18(1), pp. 35-53.

HERSHKOWITZ, R. (1990). Psychological Aspects of Learning Geometry, en Nesher, P. y Kilpatrick, J. (eds.) (1990). Mathematics and cognition: A research synthesis by the International Group for the Psychology of Mathematics Education, pp. 70-95. Cambridge: Cambridge UP.

MARALDO, S. (1980). Properties of Quadrilaterals. The Mathematics Teacher, 73(1), pp. 38-39.

VAN HIELE, P.M. (1986). Structure and Insight. A theory of Mathematics Education. Londres: Academic Press. 


\section{ANEXO I}

\section{GLOSARIO DE FAMILIAS DE PRISMAS}

Prismas rectos: Prismas con caras laterales rectángulos.

Prismas oblicuos y convexos: Prismas con caras laterales que no son perpendiculares a las bases cuya base es convexa.

Prismas cóncavos: Prismas con bases cóncavas.

Prismas de bases regulares: Prismas con bases regulares.

Prismas de caras laterales regulares: Prismas con caras laterales cuadrados.

Prismas de caras regulares: Prismas con todas las caras regulares (las bases y las caras laterales).

Prismas de caras iguales (romboedros). Prismas con todas las caras iguales (rombos iguales, incluimos los cuadrados como rombos).

Ortoedros: Prismas cuyas caras son rectángulos (incluimos los cuadrados como rectángulos).

Paralelepípedos: Prismas cuyas caras son paralelogramos.
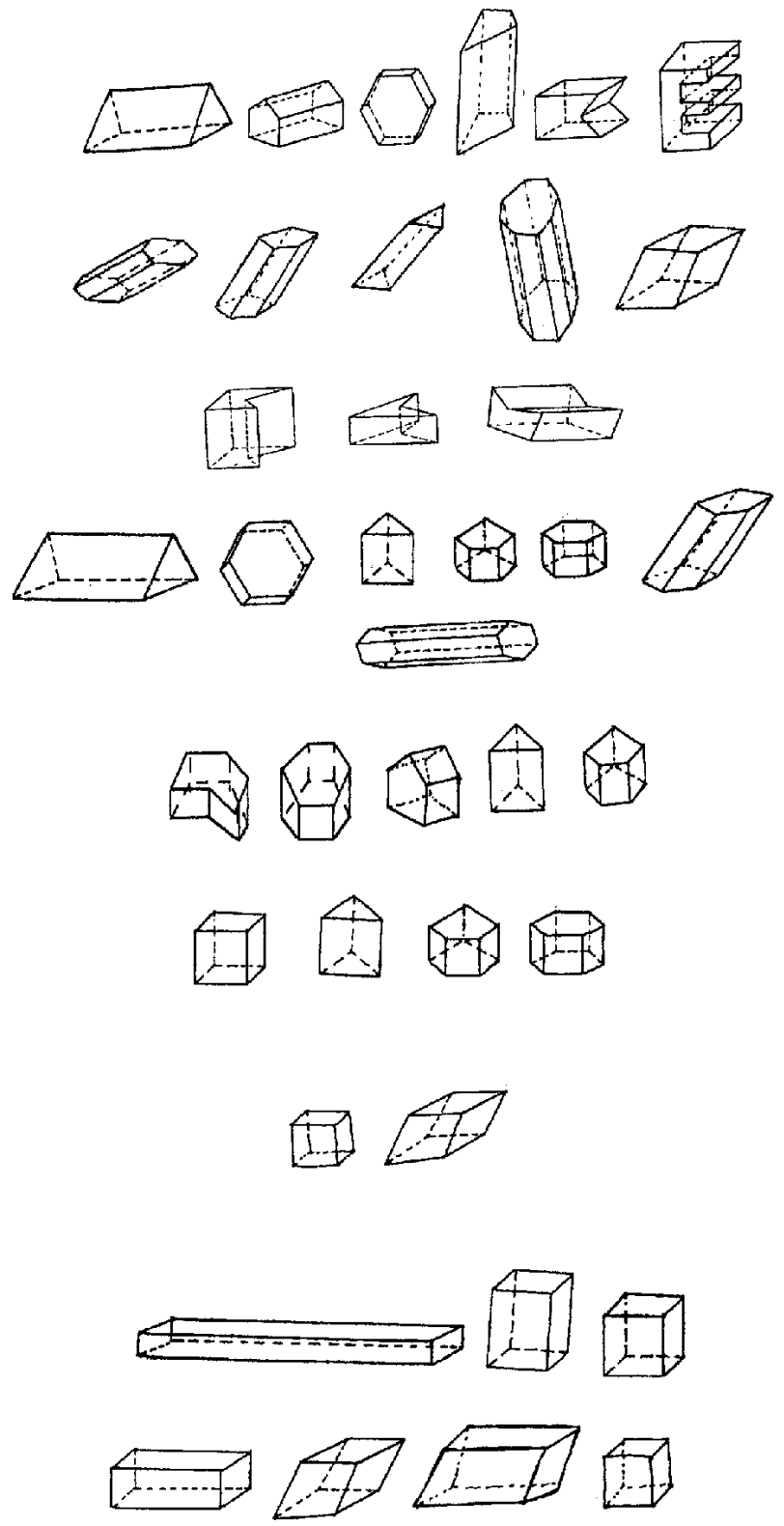

Prismas de bases trapecios isósceles: Prismas cuyas bases son trapecios isósceles. Entre ellos tenemos los ortoedros.

Prismas de bases cometas: Prismas cuyas bases son cometas. Entre ellos tenemos los romboedros.

Prismas de bases trapecios: Prismas cuyas bases son trapecios. Entre ellos tenemos los paralelepípedos. 


\section{ANEXO II}

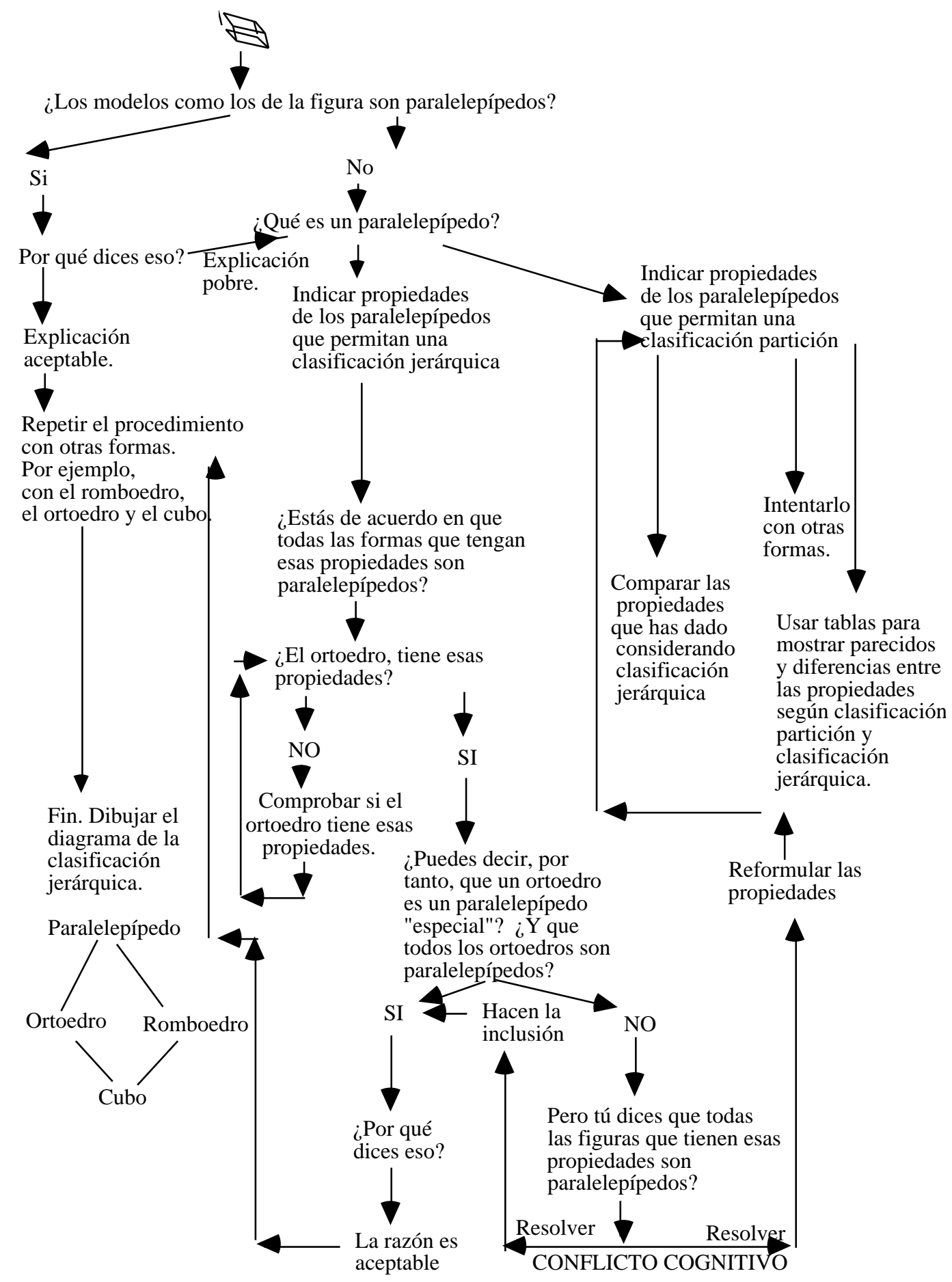

